\title{
Diamond Phononic Crystals with Silicon-Vacancy Centers at Cryogenic Temperatures
}

\author{
Graham Joe ${ }^{a}$, Cleaven Chia ${ }^{a}$, Michelle Chalupnik ${ }^{\mathrm{c}}$, Benjamin Pingault ${ }^{\mathrm{a}}$, Srujan Meesala ${ }^{\mathrm{b}}$, Eliza Cornell ${ }^{\mathrm{a}}$, \\ Daniel Assumpcao ${ }^{a}$, Bartholomeus Machielse ${ }^{c}$ and Marko Lončar ${ }^{\mathrm{a}}$ \\ a. John A. Paulson School of Engineering and Applied Sciences, Harvard University, Cambridge, MA, USA \\ ${ }^{B}$ Thomas J. Watson, Sr., Laboratory of Applied Physics, California Institute of Technology, Pasadena, California 91125, USA. \\ ${ }^{c}$ Department of Physics, Harvard University, Cambridge, MA, USA \\ grj396@g.harvard.edu
}

\begin{abstract}
We fabricate phononic crystals in single-crystal diamond hosting silicon vacancy centers. We demonstrate high mechanical Q-factors $(>10,000)$ from localized $10 \mathrm{GHz}$-frequency mechanical modes in a dilution refrigerator $(\mathrm{T}<1 \mathrm{~K})$. $\odot 2021$ The Author(s)
\end{abstract}

\section{Introduction}

Phonons, which are quantized mechanical vibrations, can be used to couple many different quantum systems [1]. Diamond is a good candidate material platform for phonon-mediated hybrid quantum systems because it has a large Young's modulus and low thermoelastic damping. This allows for high-frequency nanomechanical resonators with low mechanical dissipation. Furthermore, diamond is host to atomic impurities with optically accessible spin, which, by virtue of being located in a solid state lattice, also respond to local strain. This, combined with the large zero-point displacements present in nanomechanical resonators, which confine mechanical modes to wavelength-scale mode volumes, is predicted to allow large spin-phonon coupling rates. We study the silicon vacancy $(\mathrm{SiV})$ center, which has a large spin-strain susceptibility $(\sim 100 \mathrm{THz} / \mathrm{strain}),[2]$ positioned in a phononic crystal that supports high quality factor mechanical modes. At cryogenic temperatures, high quality factors and long spin coherence are expected to allow for quantum coherent spin-phonon interactions. Here, we present our first steps towards realizing high quality factor mechanical modes with implanted SiV center spin qubits. Specifically, we fabricate and characterize the quality $(\mathrm{Q})$ factors of $10 \mathrm{GHz}$ diamond optomechanical crystals at room and cryogenic temperatures.

\section{Methods, results and discussion}

We study an optomechanical crystal consisting of rectangular cross-section diamond nanobeams with elliptical perforations that are geometrically tapered. This taper simultaneously localizes a mechanical breathing mode near the center of the phononic crystal, at a frequency of $\sim 10 \mathrm{GHz}$, as well as a telecommunication-wavelength $(\sim 1550$ $\mathrm{nm}$ ) optical mode for readout of the mechanical excitation[3]. The waveguide region of the device terminates in a taper for adiabatic optical fiber interfacing [4]. The diamond phononic crystals in this work were designed for optimal spin-phonon coupling between the strain field of the mechanical modes and the spin qubit levels defined in a silicon vacancy $(\mathrm{SiV})$ center, based on the theory formulated by Meesala, Sohn and Pingault et al. [2].

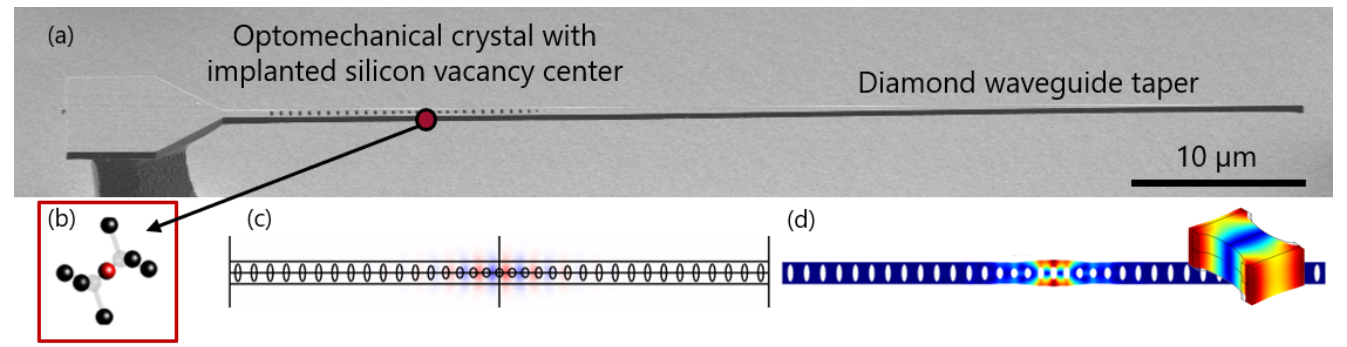

Figure. 1. (a) Scanning electron microscope image of a diamond optomechanical crystal (OMC) connected to a diamond waveguide taper. (b) Atomic structure of the silicon vacancy $(\mathrm{SiV})$ center, showing a silicon atom (red) sited between two vacancy sites (gray) in a diamond carbon lattice (black). (c) Normalized y-component electric field profile in the plane of the OMC. (d) Normalized mechanical displacement profile of the OMC localized breathing mode at $\sim 10 \mathrm{GHz}$, with the unit cell guided modes shown in insets.

To create phononic crystals with SiVs located optimally in the mechanical mode, we first pattern apertures in polymethyl methacrylate (PMMA) on single-crystal diamond using electron beam lithography, and implant silicon ions through these apertures [5]. Removing the PMMA then annealing generates SiVs at $\sim 20 \mathrm{~nm}$ below the surface where apertures were present. We then fabricate suspended phononic crystals aligned to the implanted SiVs using a 
quasi-isotropic oxygen plasma etch [6,7], in which an inductively-coupled plasma (ICP) at zero forward bias is employed to etch diamond preferentially along its crystal planes. Upon completion of the etch, the phononic crystals have a $\{100\}$ plane on its underside, thereby giving rectangular cross-section phononic crystals with SiVs positioned optimally in the mechanical mode.

The mechanical mode is optically read out by detecting the interference between pump photons, which are blue-detuned from the optical cavity resonance by an amount equal to the mechanical frequency, and scattered photons at the cavity resonance due to optomechanical coupling. The resultant thermal noise spectrum is recorded with a real-time spectrum analyzer.[8] Implanted SiV centers are optically addressed through a confocal microscope built on top of a dilution refrigerator with free-space optical access.

Optical Q-factors, measured to be $\sim 10000$, remain unchanged between room temperature and cryogenic environments. In contrast, the measured mechanical Q-factors increase from $\sim 1900$ at ambient, to $\sim 22000$ at $4 \mathrm{~K}$. However no improvement was observed upon cooling down from $4 \mathrm{~K}$ to millikelvin temperatures. This may be due to heating from the continuous-wave optical excitation used for this measurement. This is corroborated by observations of $\mathrm{SiV}$ optical spectral lines that would only be present above $\sim 1 \mathrm{~K}$, indicating that the phononic crystals are not in fact reaching millikelvin temperatures [9]. While the base temperature measured at the sample is $\sim 50 \mathrm{mK}$, we expect the device temperature to be higher owing to localized laser heating and poor thermal conductivity of the nanobeam. Confocal measurements of $\mathrm{SiVs}$ in bulk regions of the chip indicate sub-1K temperatures, further suggesting device thermalization issues. Pulsed experimental schemes can be used to mitigate the effects of laser heating, as well as devices with better thermal contact to the substrate.
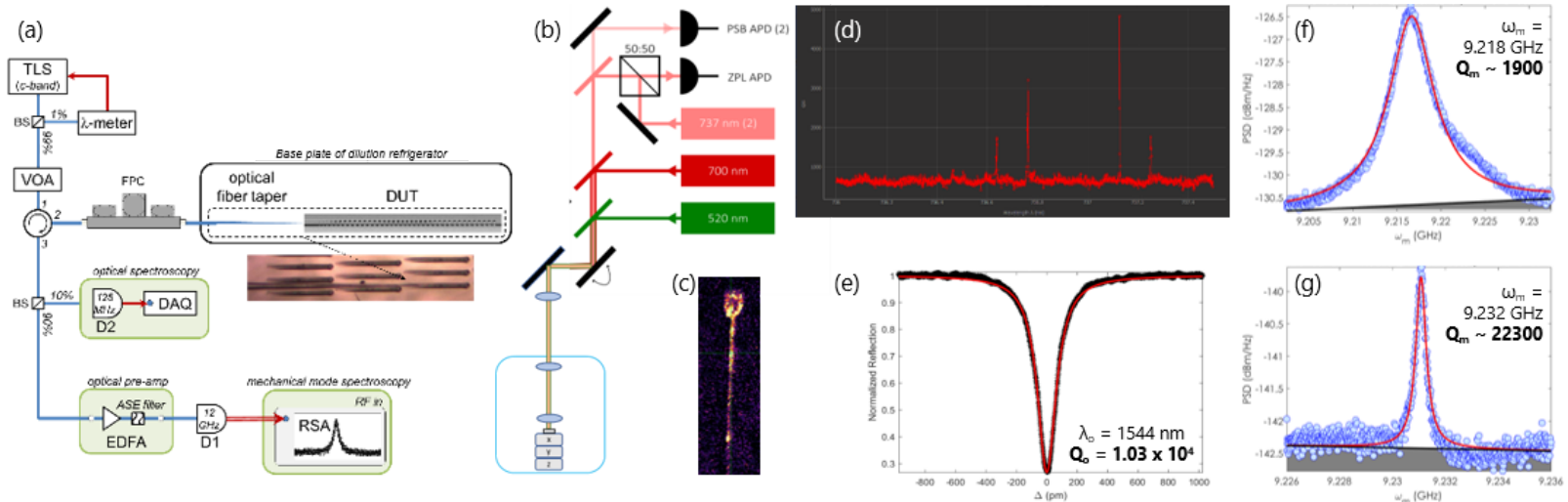

Figure 2. (a) Schematic of experimental setup for optical and mechanical spectroscopy, with micrograph showing optical fiber taper coupled to the device under test (DUT). (B) Schematic of experimental setup for confocal spectroscopy of SiVs. (c) Confocal scan of adiamond phononic crystal with SiVs. (d) Photoluminescence excitation (PLE) spectrum of an SiV. (e) Optical spectrum of the optical telecommunication mode supported by diamond phononic crystal. (f) Thermal noise spectrum of the breathing mode in ambient conditions. (g) Thermal noise spectrum of breathing mode at $\mathrm{mK}$ temperatures.

\section{References}

[1] M. Wallquist, et al.. "Hybrid quantum devices and quantum engineering." Physica Scripta, 2009 (T137), 014001 (2009).

[2] S. Meesala, Y.-I. Sohn, B. Pingault, et al. "Strain Engineering of the silicon-vacancy center in diamond.” Phys. Rev. B 97, 205444 (2018)

[3] M. Eichenfield, et al., "Optomechanical crystals," Nature, 462, 78-82 (2009).

[4] M. J. Burek, et al., "Fiber-Coupled Diamond Quantum Nanophotonic Interface," Phys. Rev. Applied, 8 (2), 024026 (2017).

[5] C. T. Nguyen, D. D. Sukachev, M. K. Bhaskar, et al., "An integrated nanophotonic quantum register based on silicon-vacancy spins in diamond," Phys. Rev. B. 100 (16), 165428 (2019)

[6] Khanaliloo, B., Mitchell, M., Hryciw, A. C. \& Barclay, P. E. High-Q/V Monolithic Diamond Microdisks Fabricated with Quasi-isotropic Etching. Nano Letters 15, 5131-5136 (2015).

[7] Mouradian, S., Wan, N. H., Schröder, T. \& Englund, D. Rectangular photonic crystal nanobeam cavities in bulk diamond. Appl. Phys. Lett. 111, 021103 (2017).

[8] M. J. Burek, et al., "Diamond optomechanical crystals," Optica, 3 (12), 1404-1411 (2016).

[9] D. D. Sukachev, et al., "Silicon-Vacancy Spin Qubit in Diamond: A Quantum Memory Exceeding 10 ms with Single-Shot State Readout," Phys. Rev. Lett., 119 (22), 223602 (2017). 